\title{
EDITORIALE
}

\section{LA VALORIZZAZIONE DELLA DIMENSIONE SOCIALE DEL MARKETING}

\author{
Valentino Gandolfi *
}

Questa nota si pone sulla scia degli ultimi editoriali di Mercati e competitività incentrati sul ruolo del marketing nello sviluppo sostenibile e sulle modalità per accrescerne la reputazione. Negli stessi sono richiamate le interessanti strategie ecologiche riscontrate in diversi casi aziendali (Frey, n. 2/2009), è indicata la guida che il marketing può tracciare per un percorso innovativo delle imprese coerentemente con le molteplici aspettative dei consumatori e con le opportunità dei mercati emergenti (Valdani, n. 3/2009), sono altresì focalizzati gli importanti apporti del marketing alla sostenibilità e al recupero di credibilità della disciplina (Cozzi, n. 4/2009). Muovendo da detti contributi, in questo editoriale si vuole portare l'attenzione sulle condizioni per la diffusione delle best practice ambientali e per valorizzare i potenziali sociali del marketing. Data la complessità della tematica e stanti i limiti di spazio consentiti, l'esposizione che segue viene necessariamente molto semplificata, mirando essenzialmente a suggerire alcuni spunti di riflessione sulla questione in oggetto.

Conformemente a tale finalità possiamo considerare primariamente le attività di marketing più visibili, quelle che compongono il marketing mix, e più in particolare quelle attinenti alla relazione prodotto-prezzo e alla comunicazione promozionale.

Circa la relazione tra le caratteristiche del prodotto e il prezzo, il marketing può senz'altro contribuire alla differenziazione ecologica dell'offerta unitamente a condizioni di prezzo accettate dai clienti e convenienti per l'impresa, ricercando e promuovendo dei segmenti di mercato composti da consumatori orientati verso beni realizzati compatibilmente con la salvaguardia delle risorse naturali, il rispetto dei diritti umani, l'equità sociale.

* Docente di Economia e gestione delle imprese presso l'Università degli Studi di Parma; vgandolf@unipr.it.

Mercati e Competitività n. 2, 2010 


\section{Gandolfi}

Diverse indagini empiriche dimostrano che nei paesi economicamente più avanzati vanno crescendo $i$ consumatori disposti a riconoscere un premium price ai prodotti con tali caratteristiche e comunque a dare la preferenza alle offerte che, a pari prezzo, si distinguono per la connotazione socio-ambientale. Gli spazi di mercato al riguardo sono rilevanti anche nel nostro Paese, ove diverse imprese hanno potuto così raggiungere eccellenti posizionamenti competitivi. Tali esempi meritano un ampio sostegno, anche perché hanno un effetto emulativo su altre imprese e perciò concorrono ad accrescere la connotazione ecologica della produzione complessiva. La quota di mercato di queste produzioni, tuttavia, continua a permanere relativamente circoscritta e l'estensione imitativa da parte delle altre aziende dei settori interessati procede molto lentamente. Pertanto, le iniziative in questione sembrano verosimilmente interessanti più sul piano aziendale che su quello dello sviluppo complessivo.

Nell'ottica aziendale preme rilevare che le azioni di responsabilità sociale dell'impresa (Rsi), come quelle leggibili in termini di differenziazione ecologica dell'offerta, devono essere valutate nel quadro complessivo delle responsabilità aziendali: la continuità temporale del conseguimento di un reddito adeguato per la remunerazione della pluralità dei soggetti coinvolti, gli adempimenti giuridici (non esclusi quelli fiscali), il soddisfacimento delle aspettative socio-ambientali connesse con un modello di consumo che tenga anche conto della qualità della vita e del benessere collettivo. Infatti, come è stato puntualizzato dalla Commissione europea (2001), "Essere socialmente responsabili significa non solo soddisfare gli obblighi giuridici applicabili, ma anche andare al di là investendo di più nel capitale umano, nell'ambiente e nei rapporti con le altre parti interessate". Per contro, è ben diffusa la qualificazione parziale della socialità delle imprese sulla base del solo rispetto di alcune normative, come quelle sull'inquinamento o sulla sicurezza degli ambienti di lavoro. Non sono poche le imprese con forti incoerenze in tal senso, che ostentano certificazioni ecologiche e sociali così impostate (anche se rilasciate da enti preposti a tali funzioni) e di fatto autoreferenziali. Interessa ancora rilevare che la valutazione della responsabilità sociale va estesa all'attività della rete di partner e fornitori di cui si serve l'impresa. Il contenuto ecologico di un prodotto avrebbe un ben scarso significato se il processo produttivo, ad esempio, fosse realizzato con l'impiego di lavoro minorile, anche dove ciò è consentito dalla legislazione vigente del paese di riferimento. Analogamente, un prodotto cosiddetto biologico, inteso come l'output di una tecnica produttiva ecocompatibile, sarebbe comunque disprezzato da un consumatore cosciente qualora fosse realizzato con lavoro in nero o lo sfruttamento di immigrati, come si è recentemente scoperto nel food made in Italy.

Le accuse al marketing riferite ad attività promozionali poco etiche per la commercializzazione dei prodotti sono frequenti e spesso fondate. Non è 
difficile imbatterci in una comunicazione non veritiera, nella promozione di consumi superflui, nella diffusione di messaggi e visioni che generano falsi valori morali. Chi fa marketing può senz'altro attenuare messaggi ed azioni commerciali del genere, ma deve necessariamente rispondere agli obiettivi assegnatigli dall'organizzazione aziendale. Tali obiettivi, essenzialmente, ruotano intorno al valore di scambio, se pur in una logica di fidelizzazione del cliente nel caso del mercato dei beni di consumo o di un mix di variabili più complesso nel caso del mercato dei beni intermedi. A questa figura risulta ben difficile optare per una politica di mercato eticamente corretta ma che non va nella direzione di massimizzare il divario tra valore percepito dal cliente e valore costo, come è atteso dai suoi vertici aziendali. Per operare "correttamente", il marketing manager dovrebbe soprattutto spingere la trasparenza socio-ambientale della comunicazione all'intero ciclo di produzione del bene da differenziare, inclusa la rete degli operatori economici esterni coinvolti. Chiaramente, si tratta di un tipo di comunicazione che implica la responsabilizzazione di tutto il management $\mathrm{e}$ in particolare del vertice decisionale. È inoltre da tener presente che la stessa efficacia di una politica di promozione dell'offerta differenziata sul piano ecologico dipende dalla reputazione etica complessiva dell'impresa, connotazione che le deriva da molteplici azioni attinenti ai tre livelli di responsabilità aziendale di cui si è detto prima.

Dunque, se si considerano le attività tradizionali di marketing non si può non rilevare che il marketing manager svolge un ruolo delimitato nell'ambito delle decisioni d'impresa, essendo condizionato dagli obiettivi del sistema aziendale complessivo e dalle finalità di chi lo governa, che non necessariamente sono permeate da quei valori morali che definiscono la Rsi nell' accezione ricordata. Molte organizzazioni aziendali tengono conto delle aspettative in questione solo quando non possono eluderle, temendo le ritorsioni del mercato, come insegna l'esperienza di note multinazionali.

La situazione cambia se non ci si limita a considerare le sole politiche di mercato. Fare marketing significa soprattutto gestire un' organizzazione market oriented volta alla creazione di valore per i clienti conformemente alle finalità imprenditoriali e alle attese degli stakeholder. Certamente, un'efficace value proposition deve essere basata sull'innovazione continua del business model, sia nel senso della sua modificazione strutturale sia nel senso di una sua ridefinizione. Al riguardo, il top management può trovare una valida base proprio nell'orientamento gestionale alla Rsi, perché a questa sono connesse vaste opportunità di innovazione individuabili nell'ambiente competitivo e nella catena del valore.

Come è stato rilevato da studiosi e da manager di diverse organizzazioni, la convenienza aziendale degli investimenti di carattere sociale va in genere attesa non nel breve periodo ma a lungo termine. Tale dimensione trova però un forte ostacolo in quel comportamento gestionale che è ormai di- 


\section{Gandolfi}

lagato nell'agire imprenditoriale, non solo nelle imprese gestite da manager. Si allude alla cultura della finanziarizzazione dell'impresa industriale, al perseguimento di risultati aziendali a breve, specie nelle società quotate in Borsa, ove gli investitori istituzionali muovono i capitali in base a rendite finanziarie e hanno imposto una nuova concezione di impresa, a cui manager e imprenditori sono indotti ad adeguarsi, in palese contraddizione con la logica implicita negli investimenti sostenibili, oltreché in quelli necessari per un effettivo sviluppo competitivo delle varie tipologie aziendali.

Per cogliere meglio la fattibilità degli investimenti socialmente responsabili è utile distinguere le diverse aree di opportunità da vagliare. Vi sono senz'altro degli interventi che elevano la qualificazione sociale dell'impresa e che danno un ritorno economico senza rischio. Si pensi, ad esempio, agli investimenti in capitale umano o in processi produttivi che risparmiano energia, i quali si configurano come azioni per accrescere l'efficienza e a volte anticipano solo un trend storico (come si rileva nel settore del risparmio energetico). Altri interventi che innalzano la dimensione sociale dell'impresa hanno invece un ritorno economico relativamente aleatorio, come rivelano le ricerche che stanno svolgendo le più importanti case automobilistiche in tema di nuovi motori, la cui convenienza è condizionata dai vincoli ambientali e dai relativi tempi di attuazione che saranno stabiliti da organismi pubblici nazionali e sovranazionali. A questi investimenti sono pertanto associati vantaggi potenziali e possibili rischi, analogamente ad ogni investimento innovativo importante. La loro valutazione dipende perciò dalla propensione all'innovazione e al rischio che caratterizza la cultura del management di una specifica impresa. Si hanno poi degli interventi che comportano un "costo aggiuntivo", come ad esempio si ha nel caso di un'impresa che rinuncia a delocalizzare la produzione in paesi a più basso costo della manodopera onde preservare l'occupazione locale. Ovviamente, l'imprenditore può decidere di accollarsi tale costo, se intenzionato a fare della filantropia, ma è evidente che una scelta di questo tipo esula dalla discrezionalità di un management che deve dar conto del suo operato agli investitori finanziari.

Nella valutazione dello sviluppo verso la responsabilità sociale occorre quindi distinguere gli investimenti che un'impresa deve comunque prendere in considerazione a seguito di imposizioni normative, o per ragioni di efficienza nell'uso di risorse naturali oppure per motivi non meno importanti come la formazione di capitale umano, da quelli "onerosi", la cui validazione economica richiede qualche forma di socializzazione dei costi, o quantomeno la predisposizione di sistemi di sanzioni/sussidi di carattere pubblico, come può essere l'attribuzione di un rating sociale alle imprese virtuose da utilizzare nelle gare d'appalto per le opere di interesse collettivo. In un contesto di mercato concorrenziale un intervento sociale che comporti un costo come quello appena indicato non può essere accettato 
nemmeno da un top management lungimirante e affatto orientato al profitto a breve, se non può contare su corrispondenti forme di compensazione pubblica. Anche il cosiddetto commercio equosolidale appare maggiormente sviluppato in quei paesi, a livello europeo, che più lo sostengono con qualche incentivazione pubblica.

Sempre in ordine ai potenziali connessi alla Rsi, è importante osservare che la loro espansione va accostata a volontà esogene all'impresa. Ad esempio, tanto più vengono abbassate le soglie tollerabili di inquinamento da parte di enti pubblici tanto più viene spinta la conseguente innovazione aziendale e, con essa, l'attività di centri di ricerca pubblici e privati. È indicativo a quest'ultimo riguardo il caso dell'automotive. In generale, le imprese sono tanto più sospinte verso l'innovazione e quindi la sostenibilità quanto più è intenso il progresso scientifico-tecnologico di base, non solo per la ricaduta sulla crescita del ventaglio delle opportunità economiche da esplorare, ma anche per gli effetti sulla riduzione del rischio da quantificare. È evidente che queste condizioni che favoriscono l'orientamento sociale delle imprese risultano strettamente dipendenti sia dalle politiche di un sistema paese sia dalle politiche delle istituzioni sovranazionali.

Il quadro operativo appena delineato, fortemente influenzato da logiche gestionali distorcenti e da decisioni istituzionali riduttive, non esclude l'importanza della ricerca aziendale di nuovi spazi di azioni di sostenibilità, che possono essere sondati soprattutto da coloro che si occupano di marketing, in virtù della peculiarità delle loro competenze. In particolare, ci sembrano da prendere in considerazione due versanti: uno proprio delle organizzazioni aziendali e l'altro, più complesso, relativo alle istituzioni pubbliche.

Circa il primo percorso è utile richiamare il tipo di rapporto tra l'impresa e l'ambiente competitivo, con cui si misura ogni modificazione del business model e in genere ogni processo innovativo. Nella letteratura economica si possono distinguere due principali approcci: uno sostanzialmente adattivo e l'altro basato sul ruolo attivo del management. Secondo il primo, l'ambiente esterno va visto come il contesto che genera sia opportunità di business che minacce destabilizzatrici, a cui l'impresa deve adattarsi. Conseguentemente, il management non può dar luogo a significativi progressi sul piano dell'innovazione connessa agli investimenti di carattere sociale, soprattutto perché questi richiedono generalmente l'intervento di soggettività costruttrici. Il secondo approccio assume invece una direzione pressoché opposta: è il management a plasmare con le sue intuizioni i nuovi business. Anche questo approccio non appare del tutto esaustivo, sottovalutando il cambiamento di origine esogena. Come è stato ben argomentato in tema di rapporto impresa-ambiente, i due percorsi unilaterali di generazione del mutamento nell'economia vanno rivisitati o quantomeno integrati con la visione interattiva, nel senso che molti potenziali macroambientali 


\section{Gandolfi}

restano tali finché non sono "inverati" da un'azione microeconomica, che in tal guisa produce innovazione e genera ulteriori opportunità di cambiamento macro e microeconomico: "Questo processo di generazione del micro tramite il macro e di generazione del macro tramite il micro costituisce nel suo insieme un fenomeno di vera e propria evoluzione del sistema industriale tramite l'interazione macro-micro" (Vaccà, Scienza e tecnologia nell'economia delle imprese, FrancoAngeli, 1989, p. 31).

Quest'ultimo approccio costituisce ora una forza determinante nel processo di elaborazione di innovazioni connesse con la Rsi e la sostenibilità, essendo queste riferite ad aspettative collettive esprimibili con il coinvolgimento collaborativo di comunità dei fruitori finali, di enti pubblici locali, di centri di ricerca, ecc. Si pensi al riguardo ai potenziali di mercato nei servizi sociali, in quelli sanitari, nei beni culturali, nel trasporto, nel settore dell'energia, ecc., valutabili e realizzabili con il ricorso al capitale relazionale, una risorsa che viene costruita soprattutto con l'azione e le conoscenze di chi si occupa della gestione dei rapporti con i clienti, della rilevazione di loro attese (anche latenti), di esperienze contestuali di consumo e del knowhy. C'è motivo di ritenere che nelle nostre imprese in generale sia poco utilizzata questa capacità di leggere la realtà sociale e di coglierne $\mathrm{i}$ potenziali emergenti per il loro sviluppo competitivo, anche a prescindere dai condizionamenti limitativi evidenziati. Questo tipo di rapporto tra le imprese e l'ambiente esterno riflette in una certa misura la stessa formazione di marketing. Nelle nostre università gli insegnamenti della disciplina sono spesso molto "manualistici", troppo preoccupati a fornire un sapere professionalizzante (ad alto tasso di obsolescenza), senza una solida base economico-manageriale, la cui carenza rischia di ripercuotersi anche sullo svolgimento del marketing tradizionale e, soprattutto, non consente al marketing manager di rapportarsi efficacemente ai bisogni nuovi della now economy.

Per quanto concerne il versante delle istituzioni pubbliche occorre muovere dall'assunto che le imprese si configurano come fondamentali attori nel processo di sviluppo sostenibile. L'orientamento gestionale alla responsabilità sociale assume però un forte impulso se le imprese si trovano ad operare in un contesto in cui sono state definite e sono fatte rispettare talune fondamentali regole economiche, ambientali e gestionali. Si può infatti dire che esiste una forte complementarità tra la responsabilità legislativa e quella sociale. Per contro, se il rispetto della normativa viene inteso da parte di imprese e organismi pubblici non un impegno civile ma un comportamento da premiare, la responsabilità sociale resta di fatto confinata in una ristretta realtà economica e viene assunta dal management come una valenza gestionale da concretizzare in un orizzonte temporale indefinito.

Ciò che viene posto in atto dalle imprese sul piano sociale è condizionato non meno dalle politiche economiche. Gli ecoincentivi decisi dal go- 
verno di un paese per promuovere l'acquisto di automobili che vengono prodotte con una tecnologia chiaramente obsoleta, ad esempio, frenano, specie in assenza di una politica industriale organica, la ricerca di nuove tecnologie e di fonti alternative di energia. Le continue dilazioni dei tempi di applicazione di limiti più restrittivi nelle emissioni di inquinanti dell'automotive decise dall'Unione europea vanno nella stessa direzione. Anche l'irresponsabilità sociale così diffusa nell'economia, leggibile nella ricerca della massimizzazione dei risultati economici a breve da parte di investitori istituzionali e quindi di azionisti, nonché di manager e imprenditori di ogni dimensione aziendale, è il portato di una serie di norme errate di carattere pubblico sul mercato del lavoro (con la conseguente dissuasione delle imprese nell'investimento in formazione di capitale umano), sulla tassazione di rendite e profitti, sulla legislazione ambientale, sull'informazione contabile delle imprese, sulla corporate governance (a cominciare dalla discrezionalità dei manager nella definizione di una performance aziendale avulsa dai risultati di lungo periodo) e così via, tutte norme in evidente contrasto sia con la logica dello sviluppo dell'impresa sia, soprattutto, con la logica della sostenibilità.

Ai fini della realizzazione di quello sviluppo sostenibile così ricorrente nel linguaggio di studiosi e politici pare quindi indispensabile un ruolo più attivo e più coerente delle istituzioni pubbliche, sia per assicurare l'applicazione della legge sia, ancor prima, per quel che concerne l'emanazione di norme volte a definire i vincoli, le opportunità, l'orientamento dell'economia: norme dalle quali dipende anche la costruzione dell'insieme dei presupposti etici condivisi, su cui poggia il buon funzionamento dell'economia. Le attività delle diverse organizzazioni economiche risultano più etiche in un contesto operativo regolato dalla giurisdizione, come insegnano gli effetti delle nuove regole predisposte dalle istituzioni americane dopo lo scandalo Enron e i comportamenti delle banche italiane successivamente alle direttive della Commissione europea circa la gestione dei tempi e delle valute di addebito e di accredito (dopo anni di inutili richiami all'etica). È ancora indicativo quanto è successo nel campo della pubblicità nella seconda metà degli anni novanta in Italia, ove è servita la pubblicazione di un centinaio di sentenze da parte dell'Antitrust per limitare la pubblicità ingannevole.

Le vicende economico-finanziarie degli ultimi decenni sembrano dunque ben rivelare l'importanza prospettica di una "diversa" regolamentazione dell'economia. Non si allude certo a un controllo di stampo dirigista ovvero di imposizioni, ma a direttive per promuovere comportamenti desiderati, a incentivi adeguati per sospingere i mercati a rispondere alle esigenze pubbliche, a provvedimenti anche per la sola correzione della de-regolamentazione prodotta dall'onda ultraliberista dell'ultimo ventennio (a cui sono riconducibili molti dei disastri causati ultimamente dalla finanza in- 
ternazionale), a condizioni volte a massimizzare la trasparenza amministrativa delle diverse organizzazioni economiche. Non si ritiene neppure che basti la regola per realizzare socialità e sostenibilità, essendo queste espressioni di valori culturali diffusi. Si è invece dell'avviso che l'auspicato progresso sociale sia la risultante della complementarità tra i comportamenti delle istituzioni e quelli della società civile, nonché tra la responsabilità legale e la responsabilità sociale.

La natura riduttiva delle decisioni prese dalle istituzioni preposte al governo dell'economia è causata in elevata misura dal comportamento generale di un potere politico fagocitato da miopi pressioni populistiche e dalle lobby delle corporazioni economiche di alcuni settori; un potere politico che si muove essenzialmente in base al ritorno di consenso senza la necessaria preoccupazione per lo sviluppo economico-sociale del paese e del mondo. Ne è un'evidente verifica, a quest'ultimo riguardo, il mantenimento delle barriere da parte dell'Unione europea nei confronti delle importazioni agricole dai paesi del Sud, la cui valorizzazione commerciale costituisce spesso la principale modalità di sviluppo delle loro economie. È dal comportamento e dall'etica di chi occupa posizioni di leadership di governo e di chi detiene il potere politico che si definisce in gran parte il sistema morale di riferimento di un paese ovvero i valori di fondo sottostanti all'agire di ogni individuo.

A ben vedere, siamo ora di fronte ad una sorta di circolo vizioso complesso che, come tale, non lascia intravedere sostanziali mutamenti in un prossimo futuro, disperde gli sforzi sociali compiuti da lodevoli imprenditori e vanifica gli stessi messaggi richiamati da Benedetto XVI in "Caritas in Veritate" per una "responsabilità sociale del consumatore", per l'adozione di "nuovi stili di vita", per "una partecipazione più sentita alla res pubblica da parte dei cittadini". Una possibile via per frangere un tale stato del sistema Italia può forse essere individuata in un' azione di "condizionamento" nei confronti dei decisori nei diversi livelli del governo del Paese, che significa contrapporre ai valori dominanti la morale del benessere collettivo e della sostenibilità, e così consolidare la condizione necessaria per il dispiegamento e lo sviluppo del cambiamento emergente a livello sociale ed economico. Di qui la proposta pretenziosa, che si vuole proporre alla riflessione del lettore, dell'elaborazione di iniziative di sostenibilità che coinvolgano più attori sociali attraverso cui diffondere siffatti valori: imprese, istituzioni pubbliche ed esponenti del mondo politico, nonchè varie espressioni territoriali e della collettività che si configurano come matrici di nuove aspettative di beni e servizi diversi da quelli che compongono l'attuale modello di consumo. Si tratta di una sfida volta a "fare cultura" o quanto meno "opinione" intorno a "progetti" con un elevato impatto potenziale sullo sviluppo del sistema economico e sociale italiano, che possono essere compresi e valorizzati essenzialmente con il ricorso a quello "stru- 
mento" dell'interazione intersoggettiva che si è ricordato prima a proposito del cambiamento della società contemporanea.

È in tale scenario che il marketing può svolgere un ruolo fondamentale nella ricerca dei segnali deboli del cambiamento e dei potenziali di creatività emergenti, traducibili in valore addizionale per la collettività coerentemente con la sostenibilità ambientale, economica e sociale. Ci si riferisce a iniziative da sperimentare tramite incontri seminariali e convegni, mirate analisi teoriche di tipo interdisciplinare, rigorose indagini empiriche, formulazione di obiettivi da perseguire, indicazione di interventi da realizzare. Si è dell'avviso che l'insieme delle competenze di marketing accademiche e manageriali che contraddistinguono la Società Italiana di Marketing (SIM) possa costituire l'innesco per esperire una siffatta intrapresa culturale di analisi e di azione, in grado di coinvolgere una pluralità di competenze e una distinta classe dirigente del Paese. La SIM potrebbe perciò spingersi oltre le basilari iniziative già svolte per interpretare la realtà economica italiana, verso una prospettiva protesa alla costruzione di un nuovo modo di produrre e di consumare, ovvero un nuovo modo di pensare e di governance. 